\title{
Fifty years ago
}

This paper, perhaps the most important ever published in the Archives, was tucked away at the very end of the 1933 volume. Fifty years later we publish in full this original description of kwashiorkor, as it appeared in the Archives of Diseases in Childhood 1933; 8: 423-33.

\section{A NUTRITIONAL DISEASE OF CHILD- HOOD ASSOCIATED WITH A MAIZE DIET \\ BY}

CICELY D. WILIIAMS, B.M.

(From the Children's Hospital, Accra, Gold Coast Colony).

There is a well-marked syndrome, not uncommonly found among the children of the Gold Coast Colony, which I have not found described. About twenty cases have been seen in the last year.

The syndrome consists of odema, chiefly of the hands and feet, followed by wasting; diarrhœa ; irritability; sores, chiefly of the mucous membranes ; and desquamation of areas of the skin in a manner and distribution which is constant and unique.

The disease attacks children of either sex, between one and four years old. It appears to be due to some dietetic deficiency and to be uniformly fatal unless treated early.

In all the cases seen there was a history of an abnormal diet. Breast feeding had been given by an old or else a pregnant woman, and the only supplementary food consisted of preparations of maize, namely arkasa and kenki, as described below.

Family customs.-In order to appreciate the salient points in the patients' history it is necessary to understand some of the local customs of the Gold Coast Colony.

African women lactate far more than European'. They breast-feed one child until the next is born, and the deposed baby is still nursed sometimes. It is rare to find an interval of less than two years between each pregnancy. The baby is, therefore, old enough to demand a varied diet-oddments from his mother's stews, vegetables, and fruit-before she becomes pregnant again. The children may be given occasional breast-feeds up to six years old and more. Not only the mother, but the grandmother, aunts, or other women living in the compound will nurse a child, not as a routine, but as a soporific in moments of stress. Some mammary secretion may be present in quite old women who have not had a child for twenty years. The grandmothers are willing to act as comforters even when there is no secrction present.

If a woman dies in child-birth or soon after, the surviving baby is generally adopted by the maternal grandmother, and breast-fed by her possibly with the help of her sisters and other daughters. The women of a family often live in a compound together, so the arrangement is simple. 
These foster-children generally die early. It is not a question of the survival of the fittest, but the survival of just a few of the luckiest. In former days, an infant that survived its mother was looked upon by the Ashanti people as a bad ' ju-ju,' and was invariably destroyed. Among the Ga and Fanti and coast tribes this is not so, but they believe that the spirit of the dead mother comes and poisons the child's food, and finally persuades the spirit of the child to follow hers.

It often happens that a child is brought to the clinic, but the medical officer is not informed that the child is motherless. The fact may be simply ignored by members of a coast tribe, or deliberately denied by an Ashanti.

To obtain a family history a very patient investigation is necessary, questions having to be repeated in two, and sometimes three, languages. With many patients to be seen, it is sometimes only by good fortune that important points in a history are discovered.

Diet.-Maize enters largely into the diet of all classes. The two forms in which it is most commonly used are called arkasa and kenki.

Arkasa is universally employed as a food for infants and invalids. It is used as a nourishing drink as we would use coffee or cocoa. In ' Gold Coast Food,' by Miss M. I. Field, the preparation is described as follows :' Soured corn-dough is mixed with water in a basin to a smooth paste, and then rubbed through a sieve into a pot so that no husks appear. More water and salt are added and the whole boiled for fifteen minutes. Sugar is often added at the end. The finished arkasa is a rather thick gruel.'

The ' corn dough' is prepared by grinding white maize, damping it, and leaving it to stand. The sour arkasa is made from corn that has been standing several days, and in which much acetic fermentation has taken place. It is often responsible for gastro-enteritis in children. The sweet arkasa is like a pap made with cornflour, and is an excellent food for children, but not by itself.

Kenki is a staple article of diet for all the coast tribes, and at all ages. To quote the same authority, it is ' corn soaked in water for three days. It is then ground on a stone, sprinkled with water, put into a pot covered with plantain leaves, and left for three days to sour. It is then ground until quite smooth; then the lump is divided into two, and one half is put into a pot of boiling water and stirred for some minutes, till thoroughly mixed with the water. The pot is taken from the fire and the uncooked half is mixed with the cooked half, and the lumps are smoothed out. The mixture is then rolled in plantain leaves or in corn trash and boiled in water for some hours.'

Arkasa, therefore, by itself or with kenki, is not likely to prove an adequate diet. It must be borne in mind, too, that white maize lacks carotene, as well as being, like red maize, deficient in tryptophane, lysine, and glycine.

The diet of the average man or woman consists mainly of kenki and other bulky carbohydrates-yams, cassava or cocoa-yams, boiled or roasted 
or beaten into a sort of purée called a 'fu-fu.' This is taken with some dried fish or a stew made of meat, tomato, onions, okra, egg-plant or beans, highly seasoned with the most fiery of peppers. Meat of goats, sheep and chickens is used only in small quantities. Beef is used seldom and only in the towns. Butter is unknown except from tins. Fats are provided mainly from palm oil, palm-kernel, cocoa-nut oil and groundnuts. Milk, apart from human milk, is not used as an article of diet. The numerous goats are never milked. Tinned milk of good brands, especially the sweetened condensed variety, is on sale in many of the villages. It has been used as a luxury for adult consumption. It is the object of the child welfare workers to have it regarded as a necessary adjunct to the children's diet. Eggs are generally only eaten when hard-boiled. As a whole, animal fats are conspicuously lacking. Fruit is eaten, but not regularly. Bananas, oranges, pineapples, paw-paws and sugar-cane can be had during most of the year; mangoes, and avocado pears in season. Tomatos, egg-plant, okra and onions are widely grown and appear in every soup or stew.

It would not be surprising, therefore, if some of the babies, though apparently healthy at birth, were lacking in some essential factors, especially those contained in proteins and animal fats. This defect perhaps only becomes obvious when there is an extra demand on the reserve stores.

History. - The history of the patients who suffer from the disease I am describing is invariably connected with improper feeding. The mother is dead and the patient has been breast-fed by a grandmother or an aunt; or the mother has become pregnant again while the patient is still young; or the mother has been ill or under-nourished. Always the story is the same-supplementary feeds have consisted of nothing but arkasa, or arkasa and kenki.

Symptoms. - This defective feeding has gone on for four to twelve nonths, and the patient has developed normally. Then he begins to get irritable; there are attacks of diarrhœa; and swelling of the hands and feet. At the end of a week to ten days the skin changes set in. If untreated, the child dies, generally within a month of the onset of the skin changes.

These changes must be described in detail. In the first place it should be remembered that an African child who suffers from a chronic disease generally shows some degree of depigmentation. This is more marked in diseases with accompanying anæmia, and is particularly conspicuous in a profound helminthiasis. A child who in health would be a rich black or a dark glossy brown, becomes gradually a dull, reddish, muddy colour. With a little experience this colour is easily distinguished from that of a child of mixed race, or that of the tribes who are naturally reddish rather than black, for instance the Fulanis, by the quality of the colour, the texture of the skin and the gloss on its surface. In the condition now under discussion, a mild degree of depigmentation takes place. The hair also in 
such a disease as a profound helminthiasis becomes dry, pale, and sparse. These changes may take place to a mild degree in this disease. There is no dryness of the skin, no branny desquamation as is described in pellagra. The changes of skin and hair are more noticeable when the disease is seen in an older child, say of four years, in whom the condition may have become chronic; or perhaps owing to occasional improvements in the diet the child has had a series of relapses and recoveries.

The early picture, then, is one of a child of about eighteen months old, well nourished but irritable, with slight œdema of the hands and feet, and the skin showing some degree of depigmentation. There suddenly appear on the ankles, on the knees, above the wrists, and on the elbows, some small black patches, first on the extensor surfaces and gradually spreading. These patches appear to consist of the epidermis which has become dark, thickened and crumpled, though it remains soft and pliable. The number and extent of these patches increase. Their distribution and their character remain distinct from the rash that is typical of pellagra. Soon the legs and forearms, knees and elbows, are covered with a sort of crazy pavement of this thickened epidermis. In a few days as the older patches mature, they strip off very readily, leaving a pink, raw surface exposed underneath. The œdema subsides at this stage. The patches of desquamation progress up the thigh and may become severe on the buttocks. Very small patches may also be seen on the face, back, and elsewhere.

The appearance of the skin condition at this stage is striking. There is the dark or reddish brown of the unaffected skin, the black patches of crumpled and thickened epidermis, and the raw areas where these have peeled off.

The skin at the corners of the mouth and eyes also begins to peel off, leaving raw areas. There is photophobia, but no eversion of the eyelid as described in the $\mathbf{A}$ and $\mathbf{B}$ deficiency of Sierra Leone.

By this time the child is in a miserable condition with sores on the mucous membranes, and perhaps a corneal ulcer. Conjunctivitis has not been marked; there has been no dryness of the cornea nor any condition resembling Bitot's spots. The child is extremely irritable, and may die in a few days if it is not treated. It appears that unless the diet is changed before the skin condition is fully developed it is impossible to save the child's life.

It may be noted that the blackened patches on the skin appear first where there is any focus of irritation or pressure. The areas round the ankles, below the knees, and above the wrists are probably determined by the strings of beads invariably worn in these positions. Over the knees, elbows, and buttocks are normal pressure points in a child who is beginning to crawl.

The irritability of these children is always great. There may also be some photophobia, but there is no alteration in any of the reflexes. Diarrhœa is persistent, especially in the later stages. The pulse is rapid, 
becoming more so as death approaches. There is an irregular type of pyrexia, which in some cases was modified by a concurrent malaria.
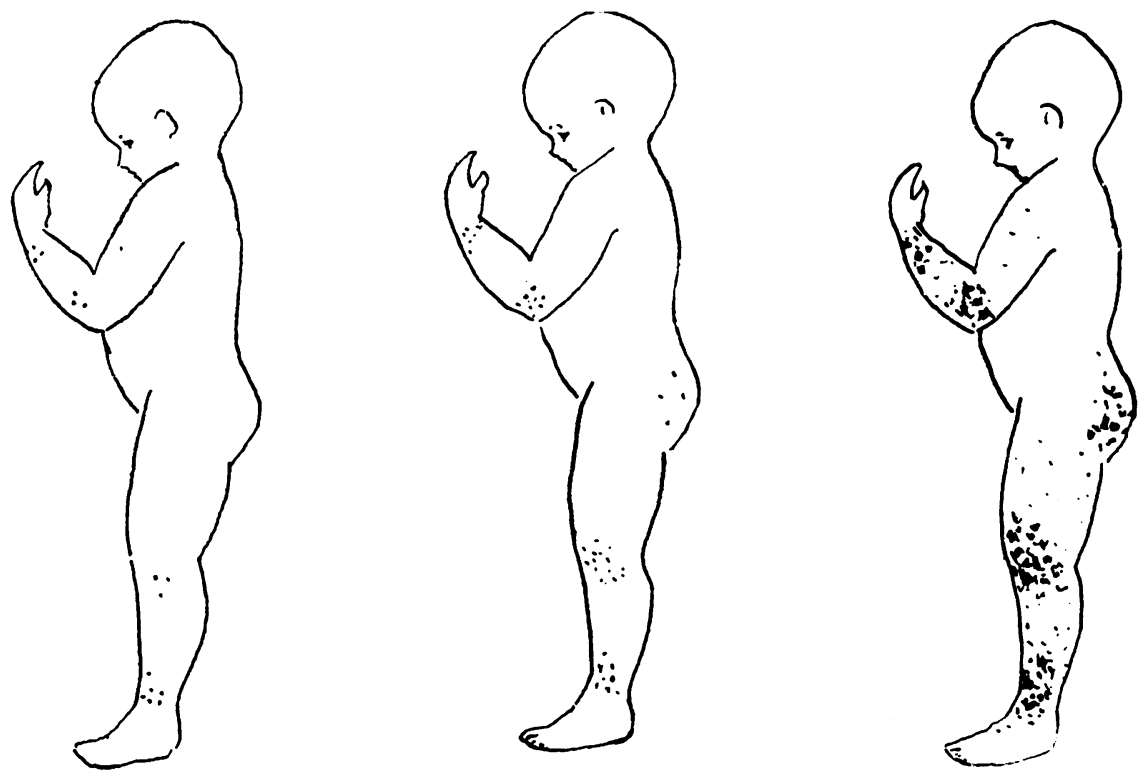

FIG. 1-3.-Showing the first appearance and spread of the rash.

Morbid anatomy.-Three post-mortem examinations have been held. All three have been exactly like that quoted at length in Case 2 , the only thing of note being a pale, fatty, almost diffluent liver.

Case records.--Five cases are given in detail. These are chosen largely because the picture was not confused by any accompanying disease.

Case 1.-Female. Her mother, a primipara, had died four days after the birth of the child. Baby was fed entirely by the grandmother, whose youngest child was said to be 2 years old at the time. The grandmother, however, looked at least 60 , and her youngest child was more likely to be 12 .

She was brought to the children's hospital fairly regularly. The fact that she was motherless was not divulged, and no special instructions were given about the feeding. She suffered from some minor ailments such as bronchitis and malaria, and she was small for her age, but until thirteen months old she grew steadily and gained weight. She then began to lose weight and showed sore mouth, acid saliva, cough, fever, swelling of feet, and intertrigo. Bowels open two or three times a day. Cod-liver oil and malt with quinine and iron, and a mouth wash, were given on July 7 th. The Wassermann reaction was negative on two occasions.

The child was brought again on July 14th. She had been so peevish that the grandmother did not get her to take the medicine regularly, and the condition was now well developed. There was much odema of the face, hands and feet. Unlike the œdema from ascaris, the cheeks were not pendulous, but there was a general puffiness all over the face. Nor was there that bulging of the eyolids so characteristic of nephritis. The tears and saliva appeared to irritate the skin, which was peeling off round the corners of the mouth and the eyes; 
The saliva was acid, there were sores in the mouth. The tears were neutral to litmus paper. The child was irritable, but all the reflexes were normal. The skin all over was atrophic in appearance; there were darker, almost pavemented patches on the forearms and the legs. The typical distribution of pellagra was absent:- the dorsum of the hands and feet seemed to escape until the last stages of the disease. The liver was enlarged, and so was the spleen. There was slight anæmia, but the blood count did not show any great abnormality. Nothing pathological was found in the stools or urine. The temperature was irregular at first, but after systematic quinine this settled down, in spite of the fact that no malarial parasites were found in the blood at this stage. In fact, except for the extraordinary condition of the skin, there were no well-marked signs or symptoms.

The dark patches on the skin became more extensive and definite; on the buttocks and other exposed parts the skin rubbed off very easily, leaving large raw areas, indolent, and healing slowly if at all.

It was difficult to examine the child's eyes on account of the irritable condition, and the fact that the gentlest handling was apt to remove large pieces of skin. After about one week the left cornea ulcerated and the eye was destroyed.

The following treatment was adopted: Iodide of iron, calcium lactate, thyroid extract, stovarsol, grey powder, and a mouth wash of sodium bicarbonate. For diet, were given: Lactogen, arkasa, eggs, paw-paw, oranges, bananas, meat-juice.

The child was in hospital for two months, and though she appeared to improve a little, the disease was never arrested.

She died on September 9th of a terminal broncho-pneumonia.

At post-mortem examination nothing definitely abnormal was found except a pale, fatty, almost diffluent liver.

Case 2.-Male microcephalic child, aged 2 years old at death.

The mother was about 14 years old when the child was born. The parents realized that the child was abnormal and neglected it. It was not brought to the hospital until 2 years old, and moribund with this disease. The parents said it was breast-fed entirely up to 18 months. The mother then became pregnant. The child was weaned and fed with arkasa only for the last two months.

The syndrome was well marked. The skin was peeling off fore-arms and legs. Liver and spleen were not palpable. The child died four days after admission.

The photograph, (Fig. 4) was taken after death.

The post-mortem report is as follows: The body was that of an ill-nourished child with a small head. Thorax, lungs and heart normal. Liver enlarged (weight, 560 grm.), pale and firm: cut surface greasy, and blood oozed from the central veins. Gall-bladder full of bile. Spleen congested. Kidneys pale : showed differentiation between cortex and medulla: capsule strips easily. Intestine: extremely thin walled; Peyer's patches prominent; contained green slimy stool.

Culture of intestinal contents; no non-lactose fermenters isolated.

Microscopic section of liver showed marked fatty degeneration.

Cause of death : ? avitaminosis.

Case 3.-Male, first seen March 29th, 1932, aged 14 months. The mother is seven months pregnant. The child was weaned four months ago; since then it has been given nothing but arkasa and kenki. It was in poor condition, with stomatitis, swelling of feet and some areas of thickened epidermis on the back.

Treatment: Cod-liver oil and malt, quinine and iron, with potassium chlorate and boro-glycerine for the mouth. Advised diet containing fruit and eggs and tinned milk, 
This treatment was not carried out, and the child was admitted two weeks later with the skin condition perfectly demonstrated. He was exceedingly peevish, with sore mouth, enlarged liver, and some cedema.

No parasites were found in the blood. Stools were loose, but showed no ova or protozoa. There was a trace of albumin in the urine. W.R. (mother and child) negative.

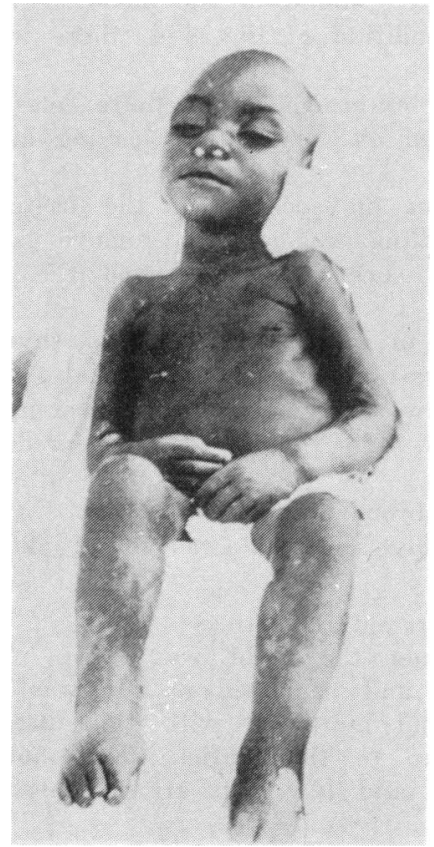

Fic. 4.-Photograph of Case 2, taken after death.

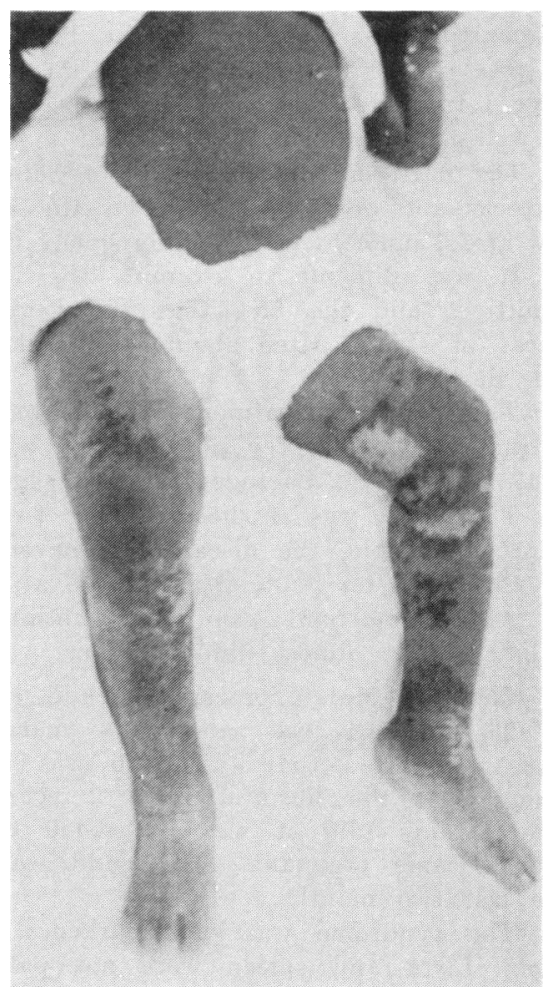

FIG. 5.-Photograph of Case 3, taken three days before death.

Treatment: olive oil to the skin; cod-liver oil and malt, and quinine and iron, with calcium lactate and parathyroid. Marmite, fruit, eggs, arkasa, milk, tomato juice, liverjuice, butter.

The condition did not improve, and on May 3rd the left cornea ulcerated. The child died on May 6th, three weeks after admission.

The photograph (Fig. 5) was taken three days before death.

Case 4.-Female, normal full-time child. When the child was 3 months old the mother became pregnant again and, at term, gave birth to twins. This child was given arkasa and breast milk. She put on weight and grew normally, except for some attacks of intertrigo and sore eyes. At 10 months old she stopped gaining weight and was ordered tinned milk, but was given practically an exclusive diet of arkasa and kenki. She was given cod-liver oil and malt, and quinine and iron when she attended the children's hospital, which was about once in six weeks. This treatment appeared to have delayed the onset of the disease. 
At 14 months old she was admitted with the disease apparent. The face, feet and hands were swollen, mouth sore, spleen and liver enlarged. Stools acid, no ova or protozoa; saliva acid to litmus. The blood contained no parasites. Reflexes normal. Purulent discharge from eyes and vagina. Irritable. There was mottling of the skin of the forearms and legs, most marked at the ankles and wrists. The skin was desquamating over these dark areas, leaving light-coloured raw patches underneath. These light-coloured patches take a long time to heal and to become normally pigmented.

Treatment: as in previous cases.

This patient appeared to recover completely while in hospital but after discharge the improvement was not maintained. When last seen at 18 months old she only weighed $15 \mathrm{lb}$. There was a return of the cedema of the face, photophobia, and irritability.

Fig. 6 shows the child convalescent at the age of 15 months. The wrinkling

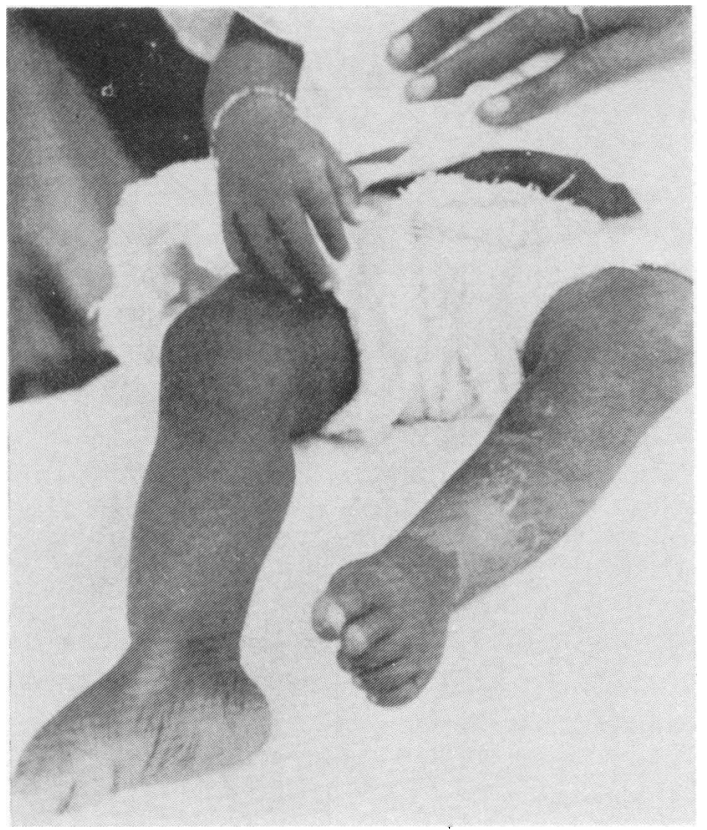

Fig. 6.-Case 4, convalescent.

on the dorsum of the feet can be seen, where the cedema is disappearing; also the dark mottling on the legs and the light coloured areas, where the new skin is showing, but has not yet become pigmented.

Case 5.-Female. The mother had had four other children. She said they had all died at about 2 years old of the same condition. She said she never had much milk, and in the present case it dried up when the child was less than a year old. She brought the child regularly to the hospital, and was given instructions about the diet, which she said were carried out. Although the child was obviously not doing well, she refused to have it admitted. Codliver oil and malt was prescribed, and it appears that this postponed the appearance of the skin lesions. When the child was about 2 years old the mother went away to the country. They returned after two months. The child showed every sign of the disease in its most profound form. After the 
child's death, the mother came one day and confessed that she had not fed her on anything but 'garri,' a Nigerian food made of cassava, arkasa and kenki. She had only once given the child any milk, and never again, as it made the child's mouth 'putto-putto' or messy.

Differential diagnosis. - The disease described above shows differences from other conditions with which it may be compared.

In pink disease œdema, wasting and irritability occur as in the present disease; but the skin lesions are quite different and the deep reflexes are lost in pink disease.

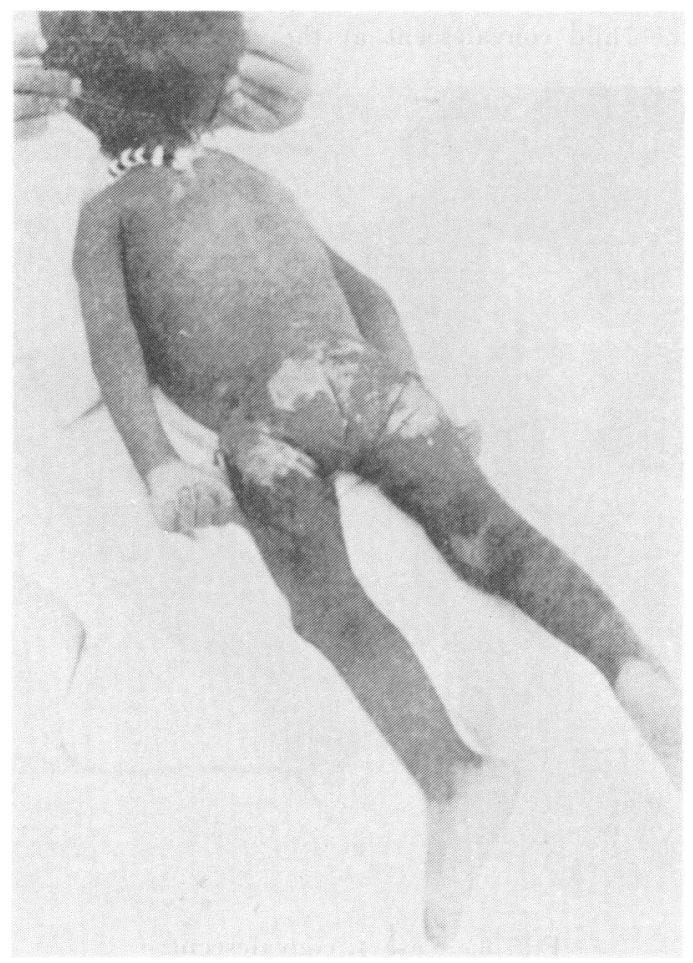

FIG. 7.

Pellagra is generally described in adults, and the character and distribution of the skin lesions are not like those described above. Similarities between the two diseases are seen in the insidious onset, wasting, mental changes, diarrhœa, and the history of a diet of maize.

In beri-beri anasarca, pericardial effusion and nervous symptoms are prominent. Skin lesions are not essential.

In pemphigus the eruption is bullous at some stage.

Treatment.-The similarity which this disease bears towards other deficiency diseases suggested that the remedy should be dietetic. In the 
absence of any accurate knowiedge as to what the defect was, a full and varied diet, rich in accessory substances, was employed, together with various drugs. In the early cases this appeared to cure, and in some of the advanced cases it appeared to arrest the progress of the disease.

It has been impossible to conduct a scientific investigation into the cause, or to make any controlled experiments into the nature of the cure. But my general impression is that the most important elements in the treatment adopted were cod-liver oil and a good brand of tinned milk. Nestlé's sweetened condensed milk seemed to answer the purpose. The cod-liver oil given to out-patients seemed to delay the onset of the fulminating stage; but it was only with in-patients, for whom an adequate supply of good milk could be assured, that a cure was established.

In three of the most recent cases attending hospital the disease was first seen in an early stage. Edema of the hands and feet was noticed, and a history of a defective diet was then elicited. In two cases the mother was pregnant, and in the third the mother had died in child-birth. Each of these children was admitted to hospital. They were given a full varied diet containing a good brand of tinned milk, eggs, marmite, fruit, cod-liver oil and malt. The cedema subsided, the irritability grew less, but the typical skin condition began to develop on the ankles. This, however, did not become serious nor did it last for long, and the general condition of the child improved rapidly. The weight increased, and in each case the child was sent out of the hospital apparently cured.

Prevention.- The young children on the Gold Coast suffer gravely from the lack of a proper diet and from the ignorance of their relations. The authorities might well actively encourage the use of milk. Merchants should be able to import reputable brands of milk, duty free if possible. The agriculturists will bestow an inestimable blessing when they will introduce a milking strain of goats to cross with the native goats. The educationalists might well devote time to teaching their pupils how to obtain milk, butter, and cheese from these animals, and include information on hygiene and feeding in the educational syllabus.

Discussion.-There are several ætiological factors which may possibly play a part in the production of this syndrome.

Breast nilk is probably deficient in some factors, which are at present uncertain.

As maize was the only source of the supplementary food, some aminoacid or protein deficiency cannot be excluded as a cause.

As regards vitamin deficiency there is no evidence pointing to lack of vitamins A, C, D or $\mathbf{E}$. That there is a deficiency of some part of vitamin B complex cannot be excluded, although the disease described here does not resemble either pellagra or beri-beri. 


\section{Summary.}

A description is given of a disease occurring in the Gold Coast Colony in children of either sex between the ages of one and four years. The disease appears to take from four to twelve months to declare itself. There is a history of deficient breast-feeding, and the only supplementary food used is a preparation of maize.

The lesions of the skin are extensive, well-marked and characteristic. They may be accompanied by slight odema of the extremities. The mucous membranes are often inflamed and ulcerated, and the saliva frequently acid. In the late stages corneal ulcers are often present. The nervous system shows persistent irritability, but the reflexes are normal.

The temperature is irregular and the pulse is rapid. There is no great anæmia, and the blood shows no leucocytosis. The Wassermann reaction is negative, and no parasites are to be found in the blood except where there is associated malaria.

There is a tendency to vomiting, and in chronic cases wasting may be severe. Diarrhuea occurs and becomes persistent in the later stages. The stools show undigested food, but no ova or parasites. The liver and spleen may be enlarged.

The respiratory system is not necessarily affected. The urine may show a trace of albumin.

Post mortem nothing characteristic is found except a very fatty, almost diffluent, liver. There may be some degeneration of the kidneys.

The treatment consists in the administration of an adequate diet rich in accessory substances.

I have to thank the staff of the Research Institute at Korle Bu for the pathological findings, Mr. Buckner for the photographs and the Hon. Director of Medical and Health Services for permission to publish these observations. I am indebted to Dr. H. M. M. Mackay and others for their help and criticism. Finally, the absence of any quotations from the literature, for which I tender apologies, is due to the fact that I am out of reach of reference libraries.

Dr Cicely Williams is alive and well and living in Oxford (where she can no longer claim a lack of library facilities). This year she celebrates her 90th birthday. We congratulate her and wish her a very happy birthday.-Ed. 